\title{
Estimating vaccination coverage in the absence of immunisation registers - the German experience
}

\author{
A Siedler (siedlera@rki.de)1, T Rieck ${ }^{1,2}$, A Reuss $^{1,2}$, D Walter $^{1,2}$, G Poggensee $^{1}$, C Poethko-Müller $^{3}$, S Reiter $^{1}$ \\ 1. Robert Koch Institute, Department of Infectious Disease Epidemiology, Berlin, Germany \\ 2. Charité, University Medical Centre, Germany \\ 3. Robert Koch Institute, Department of Epidemiology and Health Reporting, Berlin, Germany
}

Citation style for this article:

Siedler A, Rieck T, Reuss A, Walter D, Poggensee G, Poethko-Müller C, Reiter S. Estimating vaccination coverage in the absence of immunisation registers - the German experience. Euro Surveill. 2012;17(17):pii=20152. Available online: http://www.eurosurveillance.org/ViewArticle.aspx?Articleld=20152

Article submitted on 19 August 2011/ published on 26 April 2012

Immunisation registers are regarded as an appropriate solution to measure vaccination coverage on a population level. In Germany, a decentralised healthcare system and data protection regulations constrain such an approach. Moreover, shared responsibilities in the process of immunisation and multiple providers form the framework for public health interventions on vaccination issues. On the national level, those interventions consist mainly of conceptualising immunisation strategies, establishing vaccination programmes, and issuing recommendations. This paper provides an overview on sources and methods for collecting appropriate coverage data at national level and their public health relevance in Germany. Methods of data collection and available information on immunisations are described for three approaches: school entrance health examination, population surveys and insurance refund claim data. School entrance health examinations allow regional comparisons and estimation of trends for a specific cohort of children and for all recommended childhood vaccinations. Surveys deliver population based data on completeness and timeliness of selected vaccinations in populations defined by age or socio-demographic parameters and on knowledge and attitudes towards vaccination. Insurance refund claim data inform continuously on immunisation status (e.g. of children aged two years) or on vaccination incidence promptly after new or modified recommendations. In a complex healthcare system, the German National Public Health Institute (Robert Koch Institute, RKI) successfully compiles coverage data from different sources, which complement and validate one another. With the German approach of combining different data sources in the absence of immunisation registers, it is possible to gain solid and reliable data on the acceptance of vaccination programmes and target groups for immunisation. This approach might be of value for other countries with decentralised healthcare systems.

\section{Introduction}

Germany has a population of 82 million people living in 16 federal states. Health legislation with regard to communicable diseases is national and recommendations on vaccination are released annually by the German Standing Committee on Vaccination (STIKO) $[1,2]$. STIKO recommendations are not legally binding but are usually closely complied with or complemented by the official recommendations of the federal states. The official recommendation is necessary for receiving compensation in case of adverse events following immunisation. STIKO recommendations are the basis for the decision by the Joint Federal Committee (selfgoverning body of physicians and health insurance funds), whether the costs of vaccinations are covered by the statutory health insurances. In Germany, health insurance is obligatory. The majority $(85 \%)$ of people living in Germany are members of a variety of statutory health insurances, all of which provide a basic healthcare plan by statute and are funded by fixed fees paid by the members and their employers. The remaining $15 \%$ are mainly insured with private health insurance companies.

STIKO recommendations do not include specific vaccines but antigens and refer to specific target groups (defined by age, risk etc.). Beyond recommendations, all licensed vaccines can be administered, but have to be paid by the patients at their own expense.

Vaccination is voluntary in Germany. People are not only free to decide whether or not they get vaccinated but also which physician they want to consult.

Private physicians administer about $90 \%$ of all vaccinations and may choose from all available vaccines that are licensed. The remaining $10 \%$ are given in public health clinics, schools, or day care centres through special programmes of the federal states or by occupational health physicians [3]. Every vaccination has to be documented in the vaccination card of the vaccinee. Documentation includes antigen, brand name, batch number, and application date. The administering 
physician enters the same information into his patient file. Vaccination cards and files are not centrally registered.

The decentralised healthcare system together with data protection regulations which emphasise everyone's right to a self-determined use of personal data [4], are strong constraints for national registers. In only one German federal state, all vaccinations of children up to the age of seven are to be reported to the local public health services by law. However, informed consent of the parents is required prior to reporting and regulations concerning how these data should be registered and processed are lacking [5].

As vaccinations affect health at individual and population level, the assessment and evaluation of trends and of regional and demographic differences in vaccination coverage are major public health tasks. At the level of the federal states, the commitment to these tasks and their prioritisation vary and range from state campaigns and intensive monitoring of immunisation to leaving all action to the capacities and responsibility of the local health offices.

At national level, the development of immunisation strategies, the establishment of vaccination programmes, and the evaluation of recommendations are the main tasks requiring reliable and representative data on vaccination coverage. The Robert Koch Institute (RKI) as the German National Public Health Institute in the portfolio of the German Ministry of Health is responsible for collection and analysis of these data. This paper provides an overview on sources and methods used by the RKI for collecting appropriate coverage data at national level in the context of the German healthcare system and with regard to their public health relevance.

\section{Methods}

The RKI uses primary and secondary data to monitor the uptake of vaccines and to evaluate vaccination programmes. While primary data refer directly to the immunisation status as measured in, documented for or remembered by the respective individual, secondary data are obtained from sources with an original purpose different from assessing immunisation status or coverage. Primary data on vaccination coverage are gathered by the assessment of vaccination cards of children prior to school entry or by population surveys [6]. Secondary sources of information on vaccinations are data originating from physicians' refund claims from health insurances [6].

\section{School entrance health examination}

The Communicable diseases Law Reform Act (Infektionsschutzgesetz, IfSG) [7] requires to collect the vaccination status at school entry and to send aggregated coverage data to the RKI. Health examinations are carried out as a precondition for school entrance and concern children whose age varies from four to six years, depending on the federal state. The school entry cohort includes children, who should have completed their primary vaccination courses. Part of the examination is to check the completeness of recommended childhood vaccinations as documented in the vaccination card and give individual catch-up reminders where necessary. The school entrance health examinations (SEE) are carried out locally, vaccination data are aggregated at county level and sent to the federal state. Data are then transferred to the RKI once a year and include the number of investigated children, the number of children presenting the vaccination cards and the number of fully and of partly vaccinated children by defined antigens per federal state. Vaccination coverage of the respective school entry cohort is calculated based on children presenting their vaccination cards and the results, stratified by federal states, are published by the RKI in the Epidemiological Bulletin annually [8].

\section{Population surveys}

The RKI regularly conducts health examination surveys and health interview surveys, which are described in more detail below. These surveys both include crosssectional and longitudinal components in subsequent follow-ups, and are periodically carried out. The study population is geographically and socio-economically representative for the German population [9]. According to pre-defined sampling procedures, people are invited either to present to dedicated study units (for health examination and face-to-face interview) or to be interviewed by phone only (interview survey). Both forms health examination including health interview as well as health interview only - are alternately conducted.

Vaccination status is included into these surveys as one of multiple core indicators of the population's health status. It is assessed for selected antigens on the basis of either vaccination cards or reports by the subjects themselves. In the health examination surveys, data is complemented by serological testing for specific antibody.

The health examination and interview surveys for children and adolescents are conducted separately from that for adults. The nationwide representative 'German Health Interview and Examination Survey for Children and Adolescents' (KiGGS) was carried out between May 2003 and May 2006. KiGGS was based on a sample of 17,641 children and adolescents aged $0-17$ years with main residence in Germany. The 'German Health Interview and Examination Survey for Adults' (DEGS) is the respective representative survey for adults conducted by the RKI, succeeding previous adult surveys, the last of which was in the late 1990s. The DEGS was carried out from November 2008 until November 2011 in a total of 180 cities and municipalities all over Germany and included 7,988 adults aged 18-79 years. Like in KiGGS, in DEGS vaccination cards and serological tests are used to assess the immune status. 
The examination survey is periodically accompanied by the 'German Health Update' (GEDA) by waves of telephone surveys which started in July 2008 and involved already 25,000 and 23,000 people aged 18 years and over in 2008-09 and in 2009-10, respectively. Questions on vaccination status for influenza, tetanus and pertussis were included into the interviews [10].

Additional sporadic and smaller surveys for defined research questions are conducted by the RKI to gather information on knowledge, attitude and behaviour towards vaccination in the general population or in defined groups. For example, during the 2009/10 influenza season, uptake of seasonal and influenza $A\left(\mathrm{H}_{1} \mathrm{~N}_{1}\right)$ pdmog vaccines as well as acceptance and progress of the pandemic influenza vaccination campaign was monitored by consecutive representative surveys. The RKI commissioned a professional market research agency to carry out computer assisted telephone interviews (CATI) by experienced interviewers [11].

\section{Insurance refund claim data}

Statutory Health Insurance Funds are billed by the Associations of Statutory Health Insurance Physicians (ASHIP) on the basis of quarterly reports on medical services, including vaccinations, delivered by physicians. ASHIPs are regionally organised, mostly one ASHIP per federal state. In 2004, the RKI established an ongoing project together with ASHIPs using their insurance refund claim data for epidemiological assessment of vaccine preventable diseases and vaccination coverage. The methods of the project, including a detailed description of quality assurance have been described elsewhere [12]. Pseudonymised data are quarterly transmitted from the ASHIPs to the RKI and include demographic characteristics of the vaccinees (month and year of birth, sex, county of residence), information on the vaccination (vaccine, date of vaccination), and information on medical contacts (medical specialisation of physician, county of physician's office).

According to the public health question of interest, different applications of these data are possible for monitoring vaccination coverage, i.e. follow-up of birth cohorts and vaccination incidence. Information on distinct individuals can be tracked over time for receiving vaccinations. Thus, birth cohorts can be followed-up and compared by vaccination status at a certain age (i.e. 24 months) and by ASHIP region [13].

Vaccination incidence is defined as the number of vaccinated individuals in relation to the number of insured individuals of the same age and per time. Taking into account that the number of vaccinated persons accumulates over time, the cumulative vaccination incidence by a defined age and year can be calculated.

We used the insurance refund claim data and the methods described to determine varicella vaccine coverage. Varicella vaccination is part of the recommended childhood immunisation schedule since 2004. It was therefore not included in KiGGS and firstly recorded at SEE in 2008.

\section{Results}

In the following sections only selected results of the above described methods are shown as example of their feasibility and practical use.

\section{School entrance health examination}

With more than $90 \%$ in total, the vast majority of children had vaccination cards available at SEE 2009. While the percentage of children having their vaccination cards has increased over time, the range between the federal states' highest and lowest value has decreased, however the minimum was still below $90 \%$ in 2009.

SEE for 2009 showed, that vaccination coverage of children fully immunised against diphtheria, tetanus, polio (>95\% each) and Haemophilus influenzae type b (Hib, $94 \%$ ) was high [8]. Figure 1 shows the vaccination coverage for selected antigens. Immunisation gaps existed particularly for hepatitis B (90\% coverage) and for the second dose of measles (90\%). Moreover, increasing vaccine uptake was visible in recent years, especially for the second dose of vaccines containing measles antigens. Data on varicella vaccination in 2009 were available from 15 federal states and varied between $15 \%$ and $71 \%$. For almost all vaccinations, SEE-data analysis also revealed a higher coverage in eastern federal states (Saxony, Brandenburg, Saxony-Anhalt, Thuringia, Mecklenburg-West Pomerania, $n=5$ ) than in western federal states (North Rhine-Westphalia, Bavaria, Baden-Württemberg, Hesse, Lower Saxony, Rhineland-Palatinate, Berlin, Hamburg, SchleswigHolstein, Saarland, Bremen, $n=11$ ).

\section{Population surveys}

According to KiGGS, the average prevalence of completed immunisation series for tetanus, diphtheria and polio as well as the coverage for the first dose of measles vaccination was above $90 \%$ in children aged $2-17$ years. Primary courses were often not completed at the recommended age of two years. Vaccination coverage for pertussis, Hib and hepatitis B was higher in younger children than in adolescents [14]. Multivariate analyses revealed predictors for not being vaccinated or for negative serology i.e. regarding measles, such as born abroad and critical attitudes of parents towards vaccination $[14,15]$. Children not presenting the vaccination cards were more likely to be seronegative.

First results of GEDA revealed immunisation gaps for tetanus of more than $25 \%$ in the adult population [16].

About 1,000 randomly selected German speaking persons aged 14 years or older, living in private households, were included in each of the consecutive bi-weekly telephone surveys on the influenza $A\left(\mathrm{H}_{1} \mathrm{~N}_{1}\right)$ pdmo9 vaccine, during the influenza season $2009 / 10$, so that by week 53 in 2009, a total of 4,003 people had 
been interviewed. Vaccination coverage was shown to be low reaching only $8 \%$ at the end of December 2009 (Figure 2) [11].

\section{Insurance refund claim data}

For one dose of varicella vaccine, coverage at two years of age was 34\% for birth cohort 2004 and differed regionally between 13 examined ASHIPs [13]. Children of birth cohort 2005 were the first to be vaccinated with two doses of a combined vaccine against measles, mumps, rubella and varicella (MMRV) at two years of age. The varicella coverage for birth cohort 2005 was $51 \%$, including $5 \%$ of children who received two doses of MMRV. For birth cohort 2007, preliminary analysis of data from six ASHIPs showed a further increase of coverage with one dose of varicella vaccine at two years of age to $180 \%$ (unpublished data).

Annual vaccination incidence for one dose of varicella vaccine was highest in one year old children and increased here from 4\% in 2004 to $50 \%$ in 2006 (Figure 3). Cumulative vaccination incidence for the age group comprising four to six year-olds increased from $1 \%$ to $47 \%$ between 2004 and 2009.

\section{Discussion}

For the estimation of vaccination coverage at national level vaccination data as retrieved at SEE are the only routine data required by law (IfSG) in Germany [7]. Based on SEE coverage across regions can be compared and trends for complete cohorts can be assessed by all recommended childhood vaccinations. Thus, representativeness, completeness and validity are high. However, shortcomings of this approach are that timeliness of vaccination cannot be assessed at national level and that vaccinations which are newly introduced into the childhood immunisation schedule are eligible for assessment at school entry only several years thereafter. For example, since its recommendation in $\mathbf{2 0 0 4}$, varicella vaccination coverage was still not available from all federal states for the school-entry cohort 2009 for publication in 2011.

Data from SEE cover only a limited children's age group and vaccination status at other ages is not routinely collected in all federal states. Thus, they do not provide data for high interest target groups such as children at two years of age and adults for longitudinal analysis at national level.

\section{FIGURE 1}

Vaccination coverage by selected vaccinations, at school entry, in western $(n=11)$ and eastern $(n=5)$ federal states, Germany, 2002 and 2009

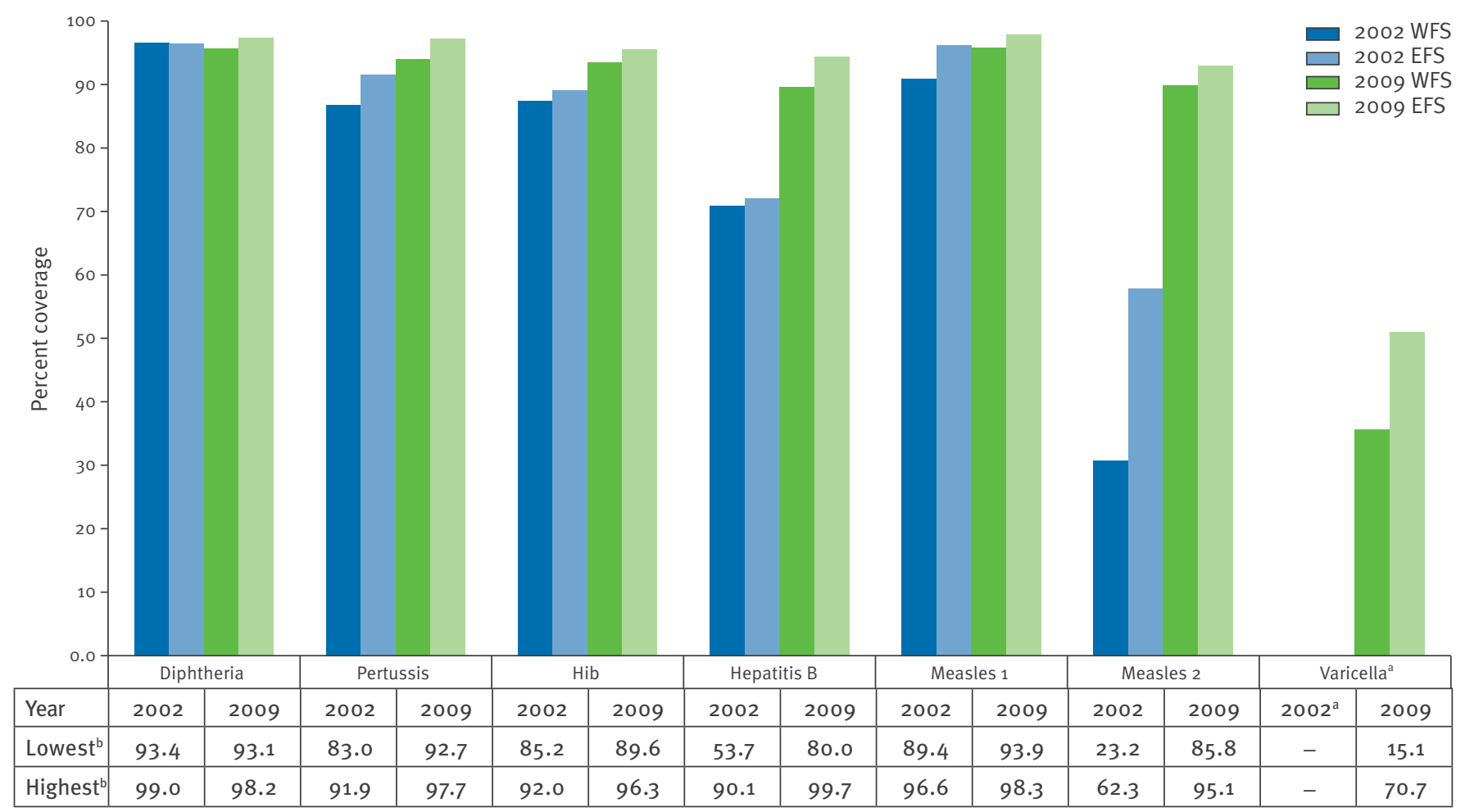

Hib: Haemophilus influenzae type b; WFS: western federal states; EFS: eastern federal states.

Vaccination coverage is given by vaccinated children per children presenting vaccination cards at school entry.

a Varicella vaccination was not yet recommended in 2002. In 2009, data on varicella vaccination were available from 15 federal states.

${ }^{b}$ Lowest and highest federal state vaccination coverage values [8]. 


\section{FIGURE 2}

Real-time monitoring of coverage with monovalent influenza $\mathrm{A}(\mathrm{H} 1 \mathrm{~N} 1) \mathrm{pdm} 09$ vaccine by countrywide telephone interviews of individuals aged 14 years or older, Germany, 16 November-29 December $2009(n=4,003)$

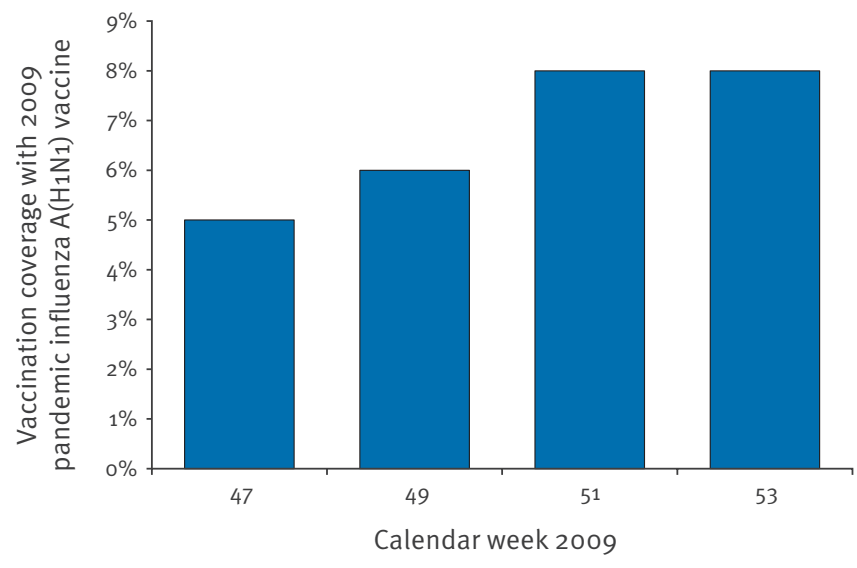

The calculated coverage from SEE based on children presenting their vaccination cards is probably overestimated. Children not presenting the cards were less likely to have been vaccinated in an outbreak investigation [17] and had a higher prevalence of seronegativity in KiGGS [18] as compared to children with vaccination cards.

Coverage as assessed by KiGGS was comparable to that of data from SEE in the same age groups, thus the respective results are validating each other. The huge benefits of examination surveys such as DEGS or KiGGS are their socio-demographic representativeness. This allows population based information on completeness and timeliness of vaccination in different groups defined by age and social status. By comparing documented vaccinations with serological results and socio-demographic parameters, risks for not being vaccinated can be predicted and essential information for composing prevention strategies is gained.

However, regular examination surveys are expensive, laborious, and complex and do not allow to estimate for recently recommended vaccinations. These shortcomings are partly overcome by telephone interview surveys. They are fast and flexible methods for collecting data and enable decision makers, for instance, to respond quickly to inadequate immunisation rates. In addition, information on perceptions on vaccination issues can easily be gained. The balance between practicability and representativeness according to the aim of the survey may lead to limitations. A general shortcoming of interviewing people for their vaccination status might be the reliability of information as compared to the documented vaccinations in official documents [19].
Insurance refund claim data may compensate the limitations of data from SEE. Moreover, if continuously collected, they can fill the gaps remaining between discrete population surveys.

ASHIP data were the only available population based source for calculating vaccination coverage by age group and region shortly after introduction of varicella vaccines into the childhood immunisation programme in July 2004. Besides a growing acceptance of the recommendation and increasing coverage particularly in the recommended age group, the data showed that a considerable amount of vaccinations were given later than recommended and that the availability of vaccines as well as the reimbursement influenced the vaccine uptake [13]. In 2009, cumulative varicella vaccination incidence for children aged 4-6 years was in the same range as coverage calculated by SEE. Moreover, based on ASHIP data the vaccination coverage for children at two years of age will be routinely assessed and indicate whether the primary courses of immunisation are completed as recommended.

ASHIP data have been available since 2004 , but vaccinations were also administered before that time, so only incident vaccinations per year since the time data collection started can be analysed. This leads to an underestimation of the true coverage, particularly in older age groups. Vaccination coverage, however, can be estimated for birth cohorts since ASHIP data collection. These data are particularly useful to monitor uptake of vaccines which are newly introduced or recommended for other age groups than before.

ASHIP data may be of limited representativeness for the total population as they are only related to statutory health insured persons, while vaccination coverage of privately health insured persons may differ. Availability, validity and reliability of coverage estimates based on data sources with an original purpose different from assessing vaccination status or coverage are highly dependent on a stable database and data structure.

ASHIP data so far have been primarily used to answer specific questions that could not be answered by other data sources. Currently, these data are being established as the official vaccination coverage of children aged 24 months in Germany. In addition, a comparison between data sources is being done to validate results whenever possible.

In summary, the described methods are of different public health relevance. Data from SEE allow to continuously analyse trends and regional differences in vaccination coverage related to childhood immunisation. With population surveys, target groups of immunisation can be defined by age or socio-demographic characteristics, and insight on the acceptance of specific vaccines can be gained. In addition, vaccination programmes and campaigns can be assessed and 
attitudes and behaviour towards vaccination examined. ASHIP data give rapid information on the compliance with vaccination schedules but also about the general acceptance of immunisation programmes, in terms of vaccination coverage by region and age, and thus are helpful for identifying target age groups for (supplementary) immunisation.

The exploitation of further primary and secondary data sources for retrieving information on vaccination coverage has been tested by the RKI with limited public health relevance.

Immunising physicians were asked for the number of administered first and second doses of varicella and measles vaccines per month in a network of private doctors (sentinel). As the sentinel is not population based, coverage could not be calculated but trends in vaccine uptake were detected and related to different physician groups, regions and availability of vaccines [20]. As the documentation workload is high, attempts are ongoing to retrieve data automatically from the software systems used by physicians, but limiting factors are the variety of those systems together with data protection.
Commercial data on sale or prescription figures deliver trends in number of sold or prescribed vaccine doses over time by brand name, region and physician's speciality. Trend comparisons by region and by physicians' speciality are possible. Moreover, with different vaccines available, insight is gained on preferred vaccine brands. These data show trends in vaccine uptake in total but not the coverage, as the number of immunised persons remains unknown. The completeness of individual vaccination series cannot be assessed, particularly if multiple doses are needed.

In conclusion, complex health systems require complex approaches to gain data on vaccination coverage or on other vaccination issues.

In the absence of immunisation registers several primary and secondary data sources have been explored by the RKI for assessing nationwide vaccination status and coverage. Different approaches for the utilisation of the various sources, either routinely or on specific demand, have been successfully implemented. Each of them has its strengths and limitations and they complement one another, thus validating the information retrieved from different sources.

\section{FIGURE 3}

One-dose varicella vaccination, annual rates for children aged one, two and three years, and cumulative rates for children aged from four to six years, Germany, 2004-2009

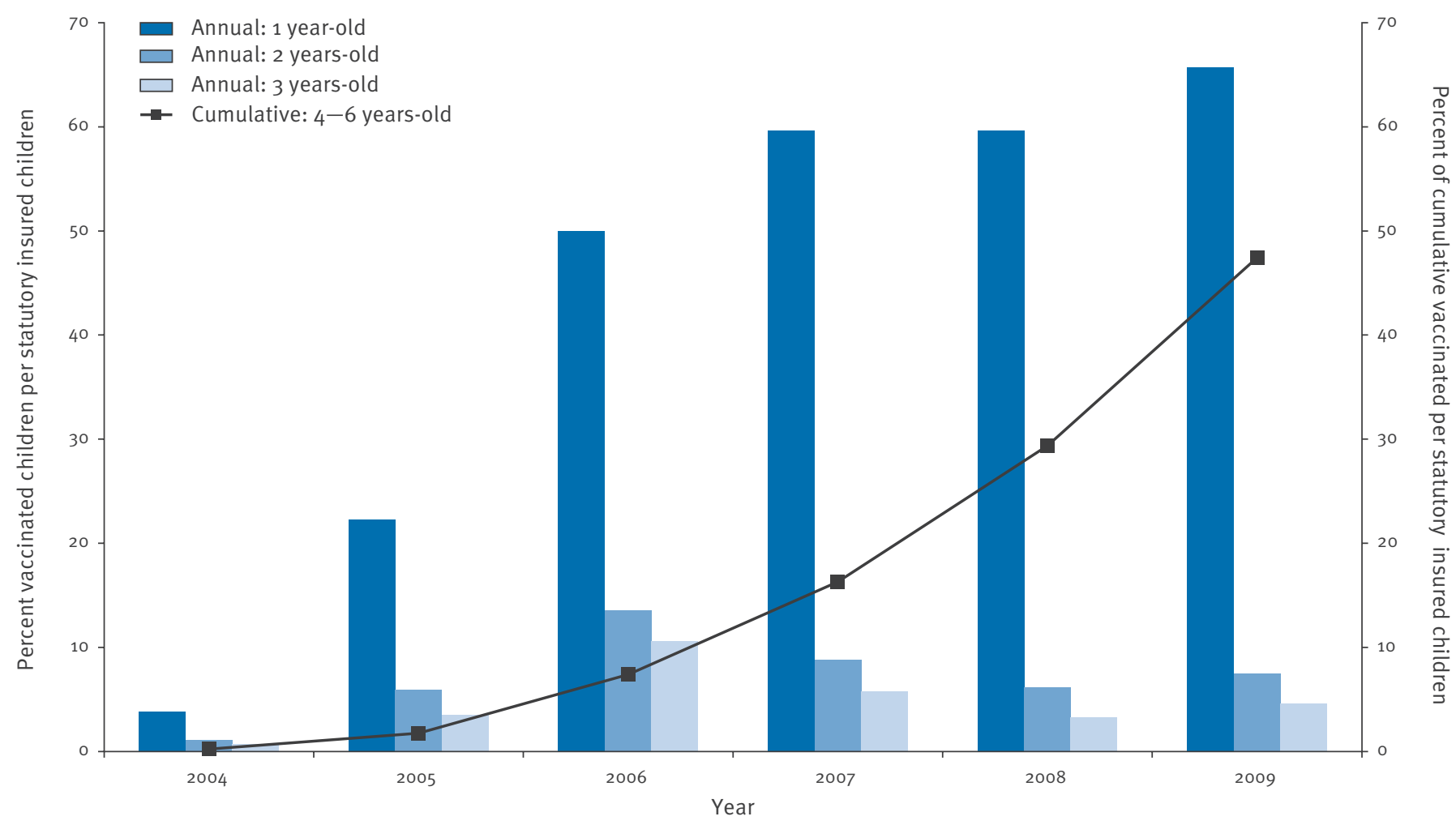

The proportion statutory insured children who are vaccinated is according to data from nine of 17 Associations of Statutory Health Insurance Physicians (ASHIP). 
The German experience with collecting vaccination coverage data at national level in a decentralised healthcare system, dominated by the private sector and in the absence of immunisation registers, might be of value for other countries with federal or otherwise decentralised healthcare systems.

\section{References}

1. Robert Koch Institut. Empfehlungen der Ständigen Impfkommission (STIKO) am Robert Koch-Institut [Recommendations of the Standing Committee on Vaccination (STIKO) at the Robert Koch Institute]. Epidemiologisches Bulletin. 2011;30:275-94.

2. EUVAC.NET. National childhood vaccination schedules Germany. [Accessed o1 Aug 2011]. Available from: http://www. euvac.net/graphics/euvac/vaccination/germany.html

3. Koch MA. Staat, Öffentlicher Gesundheitsdienst und Impfungen [State public health services and immunizations]. In: Spiess H. Impfkompendium. Stuttgart - New York: Georg Thieme Verlag; 1994. p.344-51. German.

4. Bundesbeauftragter für den Datenschutz und die Informationsfreiheit (BfDI). BfDI-Info 1.

Bundesdatenschutzgesetz. Text und Erläuterung [Federal Data Protection Act. Text and explanation]. Bonn: BfDI. 2007. German. Available from: http://www.bfdi.bund. de/SharedDocs/Publikationen/Infobroschueren/INFO1 Januar_2011.pdf?_blob=publicationFile

5. Oppermann H, Wahl G, Borrmann M, Fleischer J. [Obligatory vaccination reporting in Saxony-Anhalt. Possibilities and limitations of establishing a computerized vaccination registry]. Bundesgesundheitsblatt Gesundheitsforschung Gesundheitsschutz. 2009;52(11):1029-36. German.

6. Poggensee G, Reuss A, Reiter S, Siedler A. [Overview and assessment of available data sources to determine incidence of vaccine preventable diseases, vaccination coverage, and immune status in Germany]. Bundesgesundheitsblatt Gesundheitsforschung Gesundheitsschutz. 2009;52(11):101928. German.

7. Gesetz zur Neuordnung seuchenrechtlicher Vorschriften (Seuchenrechtsneuordnungsgesetz - SeuchRNeuG) vom 20. Juli 2000. Artikel $1 \mathrm{Gesetz}$ zur Verhütung und Bekämpfung von Infektionskrankheiten beim Menschen (Infektionsschutzgesetz - IfSG) [Act on the Reform of the Communicable Diseases Law (Communicable Diseases Law Reform Act) of 20 July 2000. Article 1. Act on the Prevention and Control of Infectious Diseases in Man (Protection against Infection Act)]. Bundesgesetzblatt (BGBl). I S. 1045. 2000. 20 Jul 2000 German. [Accessed 24 Apr 2012]. Available from: http://www. rki.de/EN/Content/Prevention/Inf_Dis Surveillance/inf dis down.pdf?_blob=publicationFile

8. Robert Koch Institut. Impfquoten bei der Schuleingangsuntersuchung in Deutschland 2009 [Vaccination coverage at the school entrance examination in Germany 2009]. Epidemiologisches Bulletin. 2011;16:125-9.

9. Kurth BM, Lange C, Kamtsiuris $\mathrm{P}$, Hölling $\mathrm{H}$. [Health monitoring at the Robert Koch Institute. Status and perspectives]. Bundesgesundheitsblatt Gesundheitsforschung Gesundheitsschutz. 2009;52(5):557-70. German.

10. Böhmer MM, Walter D, Müters S, Krause G, Wichmann O. Seasonal influenza vaccine uptake in Germany 2007/2008 and 2008/2009: Results from a national health update survey. Vaccine. 2011;29(27):4492-8.

11. Walter D, Böhmer MM, Heiden MA, Reiter S, Krause G, Wichmann 0 . Monitoring pandemic influenza $A\left(\mathrm{H}_{1} \mathrm{~N}_{1}\right)$ vaccination coverage in Germany 2009/10 - results from thirteen consecutive cross-sectional surveys. Vaccine. 2011;29(23):4008-12

12. Reuss A, Feig M, Kappelmayer L, Eckmanns T, Poggensee G. [Determination of vaccination coverage and disease incidence using statutory health insurance data]. Gesundheitswesen. 2010; 72(6):340-6. German.

13. Reuss A, Feig M, Kappelmayer L, Eckmanns T, Siedler A, Poggensee $G$. Varicella vaccination coverage of children under two years of age in Germany. BMC Public Health. 2010;10:502.

14. Poethko-Müller C, Kuhnert R, Schlaud M. [Vaccination coverage and predictors for vaccination level. Results of the German Health Interview and Examination Survey for Children and Adolescents (KiGGS)]. Bundesgesundheitsblatt Gesundheitsforschung Gesundheitsschutz. 2007;50(5-6):85162. German.
15. Poethko-Müller C, Ellert U, Kuhnert R, Neuhauser H, Schlaud $M$, Schenk L. Vaccination coverage against measles in German-born and foreign-born children and identification of unvaccinated subgroups in Germany. Vaccine. 2009;27(19): 2563-9.

16. Boehmer M, Walter D, Krause G, Mueters S, Goesswald A, Wichmann O. Determinants of tetanus and seasonal influenza vaccine uptake in adults living in Germany. Hum Vaccin. 2011 Dec;7(12):1317-25.

17. Wichmann O, Hellenbrand W, Sagebiel D, Santibanez S, Ahlemeyer G, Vogt G, et al. Large Measles Outbreak at a German Public School, 2006. Pediatr Infect Dis J. 2007;26(9): 782-6.

18. Poethko-Mueller C, Mankertz A. Sero-epidemiology of measles-specific IgG antibodies and predictive factors for low or missing titres in a German population-based crosssectional study in children and adolescents (KiGGS). Vaccine. 2011;29(45):7949-59.

19. Simpson D,Ezzati-Rice TM, Zell ER. Forty Years and four Surveys how does our measuring measure up? Am J Prev Med. 2001;20(4 Suppl): 6-14.

20. Siedler A, Arndt U. Impact of the routine varicella vaccination programme on varicella epidemiology in Germany. Euro Surveill. 2010;15(13):pii=19530. Available from: http://www. eurosurveillance.org/ViewArticle.aspx?Articleld=19530 